\title{
A nonlinear radio-photon conversion device
}

\author{
Irina L. Vinogradova ${ }^{1}$, Azat R. Gizatulin ${ }^{1 *}$, Ivan K. Meshkov ${ }^{1}$, Anton V. Bourdine ${ }^{2,3,4}$, Manish Tiwari 5 \\ 1 Department of Telecommunication Systems, Ufa State Aviation Technical University, K. Marxa street, 450000 \\ Ufa, Russia; vil-4@mail.ru (I.L.V.); azat_poincare@mail.ru (A.R.G.); mik.ivan@bk.ru (I.K.M.); \\ 2 Department of Communication Lines, Povolzhskiy State University of Telecommunications and Informatics, \\ 23, Lev Tolstoy street, Samara 443010, Russia; \\ 3 JSC "Scientific Production Association State Optical Institute Named after Vavilov S.I.", 36/1, Babushkin street, \\ St. Petersburg 192171, Russia; \\ 4 Department of Photonics and Communication Links, Saint Petersburg State University of Telecommunica- \\ tions named after M.A. Bonch-Bruevich, 22, Bolshevikov avenue, St. Petersburg 193232, Russia; \\ 5 Department of Electronics and Communication Engineering, School of Electrical and Electronics \& Commu- \\ nication Engineering, Manipal University Jaipur, J.L.N Road, Jaipur 302017, Rajasthan, India. \\ * Correspondence: azat_poincare@mail.ru
}

\begin{abstract}
The article analyzes existing materials and structures with quadratic-nonlinear optical properties that can be used to generate a difference frequency in the terahertz and sub-terahertz frequency ranges. The principle of constructing a nonlinear optical-radio converter, based on an optical focon (a focusing cone), is proposed. Based on the assumption that this focon can be implemented from the metal-organic framework (MOF), we propose a technique for modeling its parameters. The mathematical model of the process of propagation and nonlinear interaction of waves inside the focon is based on a simplification of the nonlinear wave equation. Within the framework of the developed model, the following parameters are approximately determined: the 3D gradient of the linear refractive index and the function determining the geometric profile of the focon, which provide a few-mode-based generation of the difference frequency.
\end{abstract}

Keywords: radio photonics, radio-over-fiber, orbital angular momentum, quadratic-nonlinear structure, difference frequency generation.

\section{Introduction}

One of the main strategic tasks for the development and implementation of new generation communication networks is the development of new information technologies that allow increasing the bit-rate of data transmission (up to $1 \mathrm{Tbit} / \mathrm{s}$ ) and the spectral efficiency $(60 \mathrm{bit} / \mathrm{s} / \mathrm{Hz})$ of the used radio frequency channels, as well as the use of ultrawideband communication channels (up to $100 \mathrm{GHz}$ ), high energy efficiency with ultrashort network propagation delays up to $1 \mu$ s. The solution of the set tasks can be achieved through the research and development of data processing technology based on new principles of access to the physical layer of the network, new modulation schemes and channel coding, new waveforms, improving the functioning of diversity reception technology, multiple access and security both in wireless and optical communication channels. Thus, according to the various $6 \mathrm{G}$ white papers, which regulate possible ways to implement broadband access in 6G wireless networks, it is assumed that sub-terahertz radio frequencies will be useful for the Internet of Things (IoT) and the Industrial Internet (IIoT). In these tasks, it is required to ensure the operation of non-extended radio channels, but with a significant information capacity not only between subscribers, a subscriber and a device, but also between smart devices.

Such tasks require both the use of new modulation formats and new physical layer technologies, which ensure, in particular, the "seamlessness" (absence of intermediate operations) of optical-radio conversion which is especially relevant in sub $\mathrm{THz}, \mathrm{THz}$ systems where it is very difficult to generate high-frequency radio signals by electronic devices, 
and it also seems inefficient to decode a multichannel signal for subsequent conversion of its components [1-4]. Therefore, to ensure such seamlessness, in particular, for opticalradio conversion in two-frequency (or wavelengths $\lambda: \lambda_{1}, \lambda_{2}$ ) radio over fiber (RoF) networks transmitting vortex signals (carrying orbital angular momentum, OAM), a nonlinear converter was developed [1]. But according to the results of the simulation of the converter's parameters [1] and experimental results (see Section 2), the efficacy of this converter is very low. According to our study, this is due to: a) a very small coefficient of quadratic nonlinearity $\chi^{(2)}$ of the ferroelectric crystal used in [1], and b) due to radiation losses in the specified crystal because of divergence of the input light beam, which is associated with the absence of any wave-guiding structures in the scheme proposed in [1]. The negative impact of these factors can be significantly reduced if the material and structure for the nonlinear element are properly selected. Thus, the purpose of this article is to determine the structure and the material for this type of frequency conversion.

\section{Analysis of optical quadratic-nonlinear materials and structures potentially suitable for the design of a nonlinear converter}

It was shown in [1] that during the nonlinear interaction of two input light beams in a quadratic medium, for example, in a lithium niobate $\left(\mathrm{LiNbO}_{3}\right)$ crystal, an electromagnetic beam with a frequency equal to a difference between the frequencies of the input signals is generated (difference frequency generation, DFG). In this case, several physical properties of the input beams are also transferred to the output beam (conserved), namely: for OAM carrying input beams with topological charges $\ell_{1}$ and $\ell_{2}$, respectively, at the output we obtain OAM signal with $\ell_{3}$ which is equal to the difference between the input OAMs: $\ell_{3}=\left|\ell_{1}-\ell_{2}\right|$ [1]. This fact arouses interest in the further development of the seamless principle of optical-radio conversion by a nonlinear element, presented in [1].

However, numerical estimates in [1] showed that the generation efficiency $\eta$ of a vortex (OAM) radio beam for a nonlinear medium with a length of several millimeters is extremely low (about $10^{-16}$ ). This efficiency significantly depends on the frequency ratio between the generated (difference) frequency $\omega_{3}$ and input frequency $\omega_{1}$, i.e.:

$$
\eta \approx\left(\frac{\omega_{3}}{\omega_{1}}\right)^{2},
$$

where $\omega_{3}=\left|\omega_{1}-\omega_{2}\right|$. Some causes of this were emphasized in the introduction: a small value of $\chi^{(2)}$ and the absence of a guiding structure.

If we look to the results of other authors obtained in a similar problem, we can see that this scheme is actively studied and successfully tested experimentally. Thus, in [5], the conversion of an OAM signal from the optical to the terahertz spectral region was experimentally studied using a quadratic-nonlinear element. The relations for the output signal parameters, in particular, the OAM topological charge $\left(\ell_{\mathrm{THz}}\right)$ and the conversion efficiency $\eta^{\mathrm{DFG}}$, are also obtained there, see (2) and (3).

$$
\begin{gathered}
\ell_{T H z}=\left(\ell_{O P A 1}-\ell_{O P A 2}\right) \frac{\lambda_{O P A 2}-\lambda_{O P A 1}}{\left|\lambda_{O P A 2}-\lambda_{O P A 1}\right|}, \\
\eta_{0, l}^{D F G}\left(\omega_{1}, \omega_{2}\right)=\left|\iint u_{0, l}^{L G}\left(r, \phi, \omega_{D F G}\right)^{*} u_{D F G}\left(r, \phi, \omega_{1}, \omega_{2}\right) r d r d \varphi\right|^{2},
\end{gathered}
$$

where OPA corresponds to optical signal. In this case, the conversion efficiency, according to the authors of [5], significantly depends on the beam interaction region, but the dependence on the input and output frequencies ratio, see (1), is not taken into account. The authors of $[6,7]$ also assume that a quadratic ferroelectric crystal is quite applicable for the process of parametric frequency generation: terahertz difference frequency, half-frequency, as well as double frequency and sum-frequency. In this case, the efficiency of the output beam depends on the parameters of the system and is proportional to the propagation length $\mathrm{z}$ : 


$$
I_{2}=\frac{\omega^{4} \pi^{2}}{4} \beta_{2}^{2} I_{1}^{2} z^{2} \sin c^{2}\left[\left(k_{2}-2 k_{1}\right) \frac{z}{2}\right] .
$$

The authors of [8] also present the results of experimental approbation of the considered principle of frequency transformation. According to the studies carried out, it was found in [8] that a quadratic-nonlinear crystal can indeed be used for the considered task, however, the conversion efficiency is also low - it did not exceed $0.1 \%$ of the input radiation intensity.

Having analyzed the published works in the area under consideration [5-8], our scientific team has also performed an experimental study of this principle of generation using a periodically poled lithium niobate crystal - PPLN $[3,4]$ and based on the model presented in [1], fig. 1. In this setup we used a coherent laser which signal is split into two paths and fed to two branches of free-space setup (fig. 1, b); one of the free-space branches has a diffractive optical element (DOE), forming an OAM signal; both optical signals a coaxially focused on the heated PPLN and the radio signal from its output is detected in the radio domain. It was possible to establish that the quadratic-nonlinear element used actually provides the generation of a difference frequency even when the phase matching of the waves is performed only approximately - by PPLN structure. However, the intensity of the output radio signal remains at the noise level, which significantly limits the practical usability of the considered principle. We should note that in all analyzed papers on this topic, the authors make the same conclusion: the efficiency of the difference frequency generation using this type of crystal is unacceptably low. Our theoretical approach is described in details in [1]; in any case, the results of our experiment are not the subject of this article.

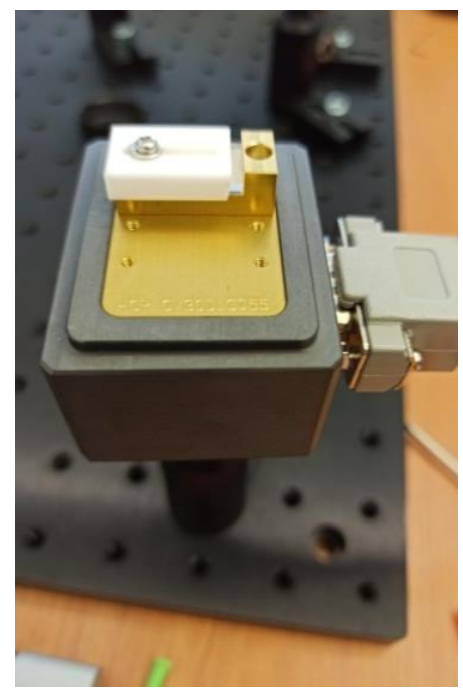

a

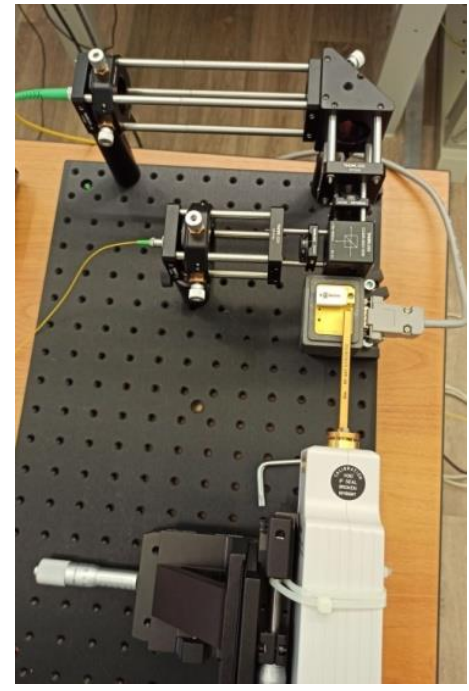

$\mathrm{b}$

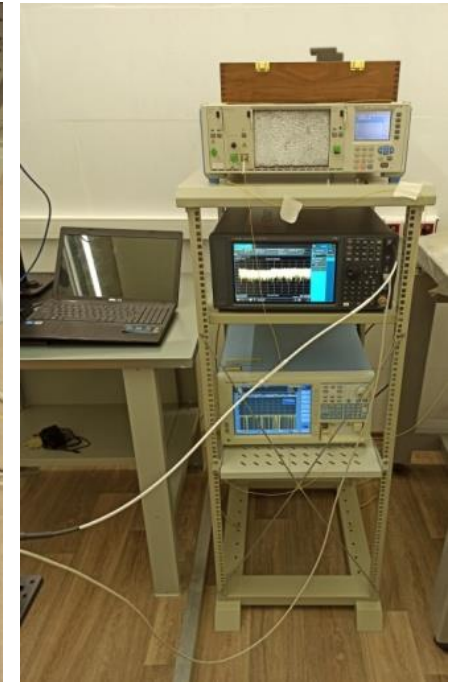

Figure 1. Photos of the fastening system $(\mathrm{a}-\mathrm{c})$ of a ferroelectric lithium niobate crystal on an experimental setup using a specialized heating interface and free-space setup (b), designed to increase the efficiency of the crystal (the heating driver is not shown; heater (a) is open); c) measurement setup including both optical and radio spectrum analyzers and a light source (laser).

At the same time, the physical reasons for this low efficiency, generally speaking, have not been studied in detail, but only assumptions have been put forward, which, moreover, differ from work to work. The general assumptions of the authors who published the results of independent studies are as follows: firstly, existing ferroelectric crystals have a small value of $\chi^{(2)}$, secondly, optical beams arriving at the input end of the crystal have a divergence, inverse proportional to the radiation wavelength. This leads to significant losses, moreover, one can list additional factors such as 1) the absence of a resonant propagation condition for the generated radio frequency; 2) violation of the few- 
mode propagation regime for the input optical radiation inside the crystal (this is in addition to the divergence). If the high-order modes are excited, the mode purity is violated and therefore efficacy decreases.

Indeed, ferroelectric crystals, such as $\mathrm{LiNbO}_{3}, \mathrm{LiTaO}_{3}, \mathrm{KNbO}_{3}, \mathrm{KTaO}_{3}, \mathrm{PbTiO}_{3}$, and the like, have such advantages as [9]: 1) low dependence of light transmission on frequency in the visible and near-IR radiation; 2) low cost and high availability. But at the same time, they have the following disadvantages: 1$)$ the value of the nonlinear susceptibility $\chi^{(2)}$, defining the efficiency of nonlinear processes, is in the range $\cong(0.5 \ldots 45) \times 10^{-12}$ $\mathrm{m} / \mathrm{V}$, i.e. is very small in comparison with other quadratic non-linear materials that exist today; 2) these materials can't be made in the form of a waveguide or fiber-optic structure, which leads to beam divergence, violation of single-mode regime and resonant propagation modes.

The small value of $\chi^{(2)}$ in ferroelectric crystals is conditioned by the physical nature of nonlinearity - it is associated with electronic polarization, and, accordingly, with the so-called electronic nonlinearity $[10,11]$. This mechanism is caused by the distortion of the outer electron cloud of atoms, ions, molecules compared to their unperturbed state, and has a very fast response time $\left(<10^{-15} \mathrm{~s}\right)$, but low $\chi^{(2)}$. Although in [12], it is shown that the listed mechanism of nonlinearity can be enhanced by spatial redistribution of electrons due to a spatially modulated light flux, which is the characteristic of spatially resonant structures, such as photonic crystal fibers. However, the materials listed above have low values of $\chi^{(2)}$, which means that they will not allow one to obtain an efficient frequency transformation.

Regarding the materials with higher nonlinearity coefficients, one can mention organic materials [13], in which the value of $\chi^{(2)}$ can be in the range $\cong 10^{-6} \ldots 10^{-7} \mathrm{~m} / \mathrm{V}$, which is relatively large. However, the mechanism for achieving such values of $\chi^{(2)}$ refers to molecular reorientation with a very big establishment time about $\sim 10^{-10}$ up to $10^{-3} \mathrm{~s}$, i.e. the greater the value of $\chi^{(2)}$ can be reached, the longer one has to wait until the material enters the non-linear regime, i.e. the longer the molecule, the greater the nonlinearity achieved, but also the longer this molecule turns. Therefore, the authors of studies in this area focus not only on the values of the coefficients obtained but also on the dynamics of the observed processes. In addition, this class of materials exhibits a significant dependence of light transmission on its physical state and condition, for example, on humidity. There is also a significant oscillating dependence on frequency in the visible and near-IR radiation.

Nevertheless, in [13] it is said that these materials can be used to generate, for example, a difference frequency in the case when this is not associated with the signal modulation but is necessary only to obtain the indicated radio emission (in other words, when one wants to generate radio carrier frequency). The authors of [13] also show that $\chi^{(2)}$ depends significantly on the ratio of the molecular axis and the electric intensity vector of the input light field, and can be found through relation (5).

$$
\chi_{k k k}^{(2)}=N f_{\text {local }} \beta_{k k k}^{\text {eff }}=N f_{\text {local }}\left\langle\cos ^{3} \theta_{k z}\right\rangle \beta_{z z z},
$$

where $\beta_{z z z}(-2 \omega ; \omega, \omega)=\frac{\omega_{e g}^{4}}{\left(\omega_{\text {eg }}^{2}-4 \omega^{2}\right)\left(\omega_{\text {eg }}^{2}-\omega^{2}\right)} \beta_{0}$.

From the point of view of the problem under consideration, in addition to the long time of the nonlinear regime establishment, a significant drawback of these materials is also the impossibility of their implementation in the form of extended waveguides or fiber optic structures.

An interesting subclass of the considered category of materials is the so-called metalorganic frameworks (MOF) [14-17]. In MOFs not only the organic filler is responsible for the macroscopic properties (see [13]), but also the metal structure, which, besides the structure strength (which was laid in the original idea of developing the MOF), can also form a field potential when irradiated with an electromagnetic field of the corresponding frequency range $[14,18]$. Strictly speaking, as we know, there are no results of experimental studies on the effect of the metal frame that makes up the MOF on the possible 
generation of plasmon field effects, which affects the macroscopic optical properties of the material. It should be noted that the nonlinear properties of MOF structures are studied quite intensively in both theoretical [19] and experimental [20] ways. For example, in [21], research is primarily focused on the design of a molecule that provides the required nonlinear properties.

According to publications [15, 17], MOFs can be used to form an optical quadratic nonlinearity in problems of generating, for example, the second harmonic:

$$
I_{2 \omega}=\frac{32 \pi^{3} \omega^{2} s^{2} \theta_{2 \omega}}{c^{3}}\left|e_{2 \omega} \chi^{(2)} e_{\omega}^{2}\right|^{2} I_{\omega}^{2} \infty\left(\chi^{(2)}\right)^{2} I_{\omega}^{2} .
$$

The main properties of MOFs from the point of view of the problem under consideration include:

1) low dependence of light transmission on frequency in the visible and near IR region,

2) the $\chi^{(2)}$ is in the range $\cong 10^{-3} \ldots 10^{-9} \mathrm{~m} / \mathrm{V}$, which is by several orders of magnitude higher than in ferroelectric crystals,

3) non-linear regime establishment time is $\sim 10^{-14}$ up to $10^{-3} \mathrm{~s}$ (the spread is very significant because the time depends significantly on the influence of the metal frame),

4) the properties of MOF can be controlled by an external field/potential,

5) MOFs cannot be made in the form of extended guides/fiber optic structures.

In the framework of the considered problem, MOF under certain conditions can be used as the basis of a quadratic-nonlinear element. Namely, in the case of a decrease in the nonlinear regime establishing time (to values of $10^{-14}-10^{-13} \mathrm{~s}$ ) due to the use, for example, of plasmon effects.

Another option for constructing a quadratic-nonlinear element with properties suitable for the problem being solved is the use of a photonic-crystal fiber (PCF). It should be mentioned that PCFs have the widest range of macroscopic optical properties: in some cases, their nonlinearity is reduced to zero due to the propagation of the light field in areas filled with air [12]; in other cases, they obtain a significant macroscopic nonlinearity [12, 22-27] achieved, for example, by channeling the light field in regions of ultra-small diameter, which provides a significant increase in the intensity and a corresponding increase in the effective nonlinear response. As an example, this option is used for spectrum broadening by the interaction of nonlinearity on short pulses (with durations $\sim 10^{-12} \mathrm{~s}$ ).

The results concerning the quadratic nonlinearity in PCF have been published widely [23-27]. So, in [25] it is said that $\chi^{(2)}$ can be in the range $\cong 10^{-3} \ldots 10^{-10} \mathrm{~m} / \mathrm{V}$, which many times exceeds the corresponding values for ferroelectric crystals. There are additional mechanisms for obtaining nonlinearity $[23,24]$ associated with injected carriers, which are put into operation with a nonlinear shift of the photonic bandgap [23]. At the same time, this mechanism is inherently akin to electronic nonlinearity, which means that it is characterized by a short establishment time. Therefore, in quadratic-nonlinear PCFs constructed in the above manner, one can expect significant $\chi^{(2)}$ along with short entry times into the specified regime. As we know, there is no published data on the dynamic parameters of the nonlinear regime of PCF. It is also obvious that PCFs are waveguiding structures, which is undoubted of interest for the problem being solved.

It is mentioned in $[26,27]$ that quadratically nonlinear PCFs can be successfully used to generate the second harmonic and difference frequency [28], including the $\mathrm{THz}$ range [29]. But if a high nonlinearity is achieved by spreading the input light beam over highintensity narrow light "filaments", then this will actually lead to multipole [30] generation of the radio frequency wave and, quite possibly, to distortion of the information signal carried by it. Therefore, PCFs can potentially be used for the problem of square-nonlinear generation of the RF difference frequency, but the considerations presented above will require optimization of the PCF structure to reduce the distortion of the generated signal, as shown in the above-mentioned papers.

Thus, according to the above analysis of materials and structures with quadraticnonlinear optical properties, it can be concluded that MOFs and/or PCFs can be used for 
the problem being solved. In the first case, i.e. for MOF, one can either use the parameters of some known structure (material) to develop a non-linear element for DFG or study the effect of a metal frame and thereby optimize MOF parameters. It is quite possible that in the latter case, in order to form the desired optical properties (e.g., to control the time of nonlinear regime establishment) it may be necessary to use additional radiation $\left(\lambda_{\text {pump }}\right)$, fig. 2, which shifts the operating point of the non-linear regime and thereby catalyzes the process. In the second case, i.e. for PCFs, it is necessary to take into account the properties of the photonic-crystal structure that determine the mechanism of nonlinearity and, accordingly, the mechanism for generating a radio wave, and generally speaking, to optimize the $\mathrm{PCF}^{\prime}$ s nonlinearity for the problem under consideration.

\section{Proposed conversion device and formulation of the problem of modeling its param- eters}

As can be seen from the analysis above, the simplest solution is the use of MOF, as presented in [17]. In this regard, to minimize losses and increase the efficiency of a radiophotonic conversion device designed to generate a radio frequency, it is necessary to make a nonlinear element in the form of a guiding structure that retains resonant properties for both input optical and output radio radiation. This can be done by changing the diameter of said guide structure $D(z)$ along the propagation axis $z$ as in horn antenna or focon. In this case, for the input optical radiation $\left(\lambda_{1}, \lambda_{2}\right)$, it is desirable to maintain a (quasi)singlemode propagation over the entire length $L$ of the nonlinear element. Or, in any case, to optimize the parameters of the nonlinear element so that the single-mode propagation mode for the input radiation is maintained as long as possible. This can be achieved by changing the radial profile of the refractive index of the material along the length of the nonlinear element, i.e. providing the corresponding refractive index profile $n(z, r)$. Note that by "(quasi)single-mode" we mean the OAM mode (within the scalar approximation it corresponds to a set of linear polarized (LP) modes containing angular exponent $\exp (i \ell \varphi)$, and each of these modes is a superposition of degenerated transverse modes [31]), so generally speaking, we are talking about few-mode regime.

Thus, the proposed radio-photonic conversion device should have the following structure, fig. 2. Optical radiation is supplied to its input at two close wavelengths, the difference of which corresponds to the wavelength of the generated radio wave. Both input optical wavelengths may be fed through a single input optical fiber. One should use a few-mode fiber (FMF) if the optical wavelengths lie in C-band. In the case when it is necessary to use additional (corrective) radiation $\left(\lambda_{\text {pump }}\right)$, the input optical channel can be implemented, for example, by a two-core concentric optical fiber, fig. 2 . The output part of this radio photonic device must have a substantially larger diameter than the input part, which is necessary to ensure effective resonant properties for the generated radio wave. Since the relationships that interconnect the geometric parameters of the guiding structure with the mode wavelength are linear, we can estimate:

$$
\frac{D_{\text {out }}}{D_{\text {in }}} \approx \frac{\Lambda}{\lambda},
$$

where $\Lambda$ is the wavelength of the generated radio wave; $\lambda$ is one of the input optical wavelengths.

Therefore, we can consider the nonlinear element as a focon, whose geometric profile $D(z)$ can either be a continuously increasing function or have a minimum (as shown in fig. 2 ) in the case of a significant inlet diameter. Such a focon can be made by MOF. The design of the focon parameters is reduced to finding $D(z)$ and $n(z, \mathrm{r})$ with known $\lambda_{1}, \lambda_{2}, \chi^{(2)}, D_{\mathrm{in}}$, $D_{\text {out, }}$ and focon length $L$ provided that the amplitude of the output radio wave $A_{\Omega}$ is above a given threshold $A^{*}: A_{\Omega} \geq A^{*}$. An additional physical condition that ensures $A_{\Omega} \geq A^{*}$, as mentioned above, is the requirement to maintain a quasi-single-mode regime for input radiation at length $H$, which in the limit $H \rightarrow L$. Note that if the focon is made of the PCF, the parameters of the PCF structure should also be included in initial design parameters since the PCF's nonlinearity cannot be replaced by the averaged $\chi^{(2)}$. 
Geometric profile of the focon $f_{1}(\mathrm{z})$

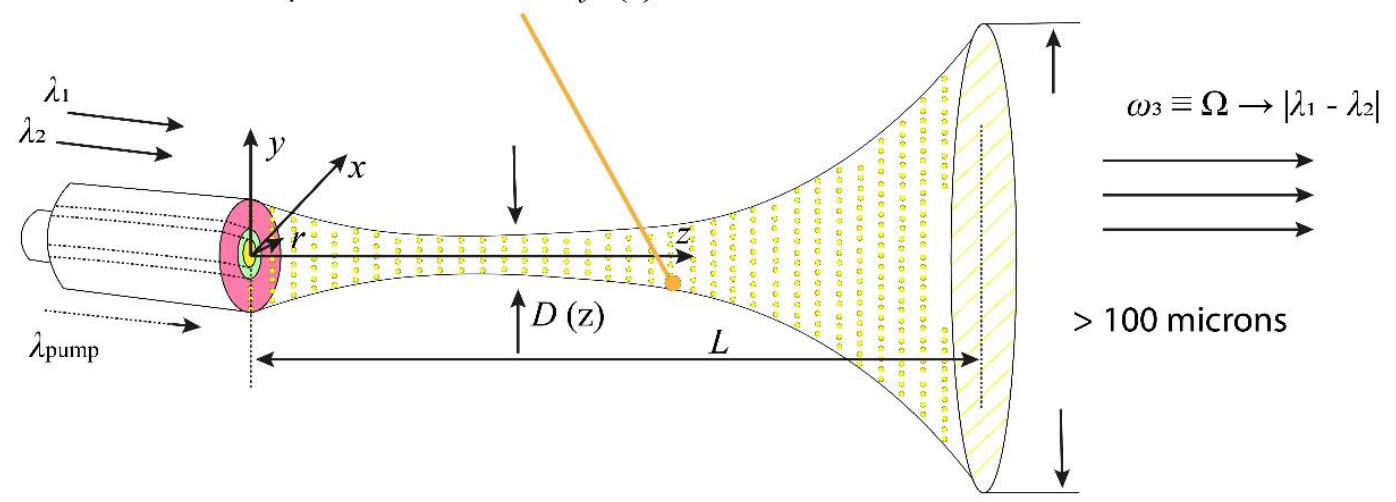

Figure 2. An illustration of the structure of the proposed radio-photonic conversion device that provides the generation of differential radio signal when optical radiation is supplied to the input at two close wavelengths.

Based on the above, the problem is posed of modeling the magnitude of the electric field strength for the input (with indices 1 and 2) and output (3) waves to determine the spatial distribution of the refractive index of the focon and its geometric profile. We introduce the following parameters:

- for the focon profile one can write: $x^{2}+y^{2}=r^{2}=f_{1}(z)$, let $f_{1}(z)$ be a smooth function;

- $n(z, r)$ is the linear refractive index for $r^{2}<f_{1}(z)$ inside the focon. In this case, we will assume that $n(r, z)=n_{0}+\Delta n(r, z)$, and $\Delta n(r, z)$ determines the gradient of the refractive index, and in relative representation $\Delta n(r, z)$ is the same for each of the waves: $n(r, z)=n_{0}+\theta(r, z) n_{0}=n_{0}(1+\theta(r, z))$, where $n_{0}$ is initial (unperturbed by the gradient) refractive index. Thus, the undetermined function for all waves is $\theta(r, z)$, and, as the results of similar studies presented in [32-35] show, $\theta(r, z)$ most likely will not exceed a few percents of $n_{0}$. For definiteness, we set: $|\theta(r, z)| \leq 0,1 \cdot n_{0} \theta(r, z)$. Note that the function $\theta(r, z)$ must satisfy some obvious requirements, for example, it must be continuous and monotonic; such requirements are dictated by the condition of focon's physical realizability. In this way, $n(r, z)=n_{0}+\Delta n(r, z)=n_{0}+\theta(r, z) n_{0}$.

- the parameter $\chi^{(2)}$ can be considered homogeneous over the focon volume, since it changes slightly in the presence of a linear refractive index gradient. This assumption is due to the fact that the effect of the gradient of the linear refractive index on the phase of the signal during the interference process is much more significant than the effect of a change on the nonlinear process (see below). In other words, when the linear part of the refractive index changes, its nonlinear part undergoes proportional changes, which is, however, very insignificant. We also neglect the dispersion of $\chi^{(2)}$ for the considered waves according to [36].

- refractive index outside the focon $n(r, z)=n_{\mathrm{cl}}$ for $r^{2}>f_{1}(z)$, where we assume that $n_{\mathrm{cl}}=1.46$ and index "cl" stands for "cladding".

\section{Modeling the parameters of the proposed device}

The propagation of an electromagnetic wave in a nonlinear medium is described by the following equation:

$$
\nabla^{2} \vec{E}-\frac{\varepsilon}{c^{2}} \frac{\partial^{2} \vec{E}}{\partial t^{2}}=\frac{1}{c^{2}} \frac{\partial^{2} \vec{P}_{N L}}{\partial t}
$$


where $\vec{E}(x, y, z, t)$ is the vector of the electric field strength, $c$ is the speed of light in vacuum, and $\varepsilon$ is the permittivity of the medium, and $\vec{P}_{N L}(x, y, z, t)$ is the nonlinear polarization vector.

By analogy with [1] and using the technique described in [36], to obtain a solution (8), one should, firstly, use the separation of variables, and secondly, apply the Fourier transform of the given functions in the time domain. The latter allows in (8) to get away from time derivatives by performing the following replacement: $d / d t \rightarrow j \omega$. So, the Fourier image of the solution (8) will be written in the form:

$$
\tilde{E}(x, y, z, \omega)=\tilde{A}(z, \omega) \tilde{F}(x, y) \exp (j \beta z) \equiv E(r, \varphi, z, \omega)=A(z, \omega) F(r, \varphi) \exp (j \beta z),
$$

where we omit the symbol $\sim$ in the further notation for simplification. Now we change the Cartesian system to cylindrical coordinates (we consider the focon to be a body of rotation); $\omega$ is the radiation frequency, $\beta=\frac{\omega}{c} \sqrt{\varepsilon}, A(z, \omega)$ is a function describing the longitudinal (along the focon, fig. 2) dependence of $E$, and $F(r, \varphi)$ is the transverse component of the in $E$. In addition, in (8) and (9) the indices 1, 2 and 3 related to the input (1 and 2) waves and the output (3) wave, respectively, since the above expressions for all three waves appear to be identical.

Next, we find the derivative of (9) with respect to coordinates, and substitute the resulting relation into (8), taking into account the properties of the Fourier transform. Following [35], we neglect the second derivatives with respect to coordinates to obtain the final equation, since we assume that functions $A(z, \omega)$ and $F(r, \varphi)$ change much slower than $e^{i \beta z}$.

Now, if we divide $\beta$ into components depending on the basic physical processes under consideration, we can obtain two equations of the form:

$$
\frac{d A}{d z}=j\left[\beta_{\Phi}(\omega)-\beta_{0}\right] \cdot A \text { and } \frac{d F^{(q)}}{d r}=j\left[\beta_{\Phi}(\omega)-\beta_{0}\right] \cdot F^{(q)},
$$

where $\beta_{\Phi}(\omega)$ determines the additional phase incursion of the wave under the influence of the determining physical process. So, for example, if a light wave propagates in an extended optical path, then the determining physical process will be chromatic dispersion. In this article, we assume that there are no dispersion distortions during the propagation of waves inside the focon since we consider two close wavelengths. On the contrary, significant influence have quadratic-nonlinear change in the refractive index, characterized by $\chi^{(2)}$, when the waves propagate along the focon, and the interference of waves during transverse distribution, which ensures the addition of re-reflecting waves into a single mode. But the function $F$ in (9) also has a $z$-axis dependence, moreover, as shown in [3234], to obtain a proper solution one should divide the entire focon into transverse layers of width $\Delta z$ where $\Delta z \rightarrow 0$, fig. 3. Within each layer, the "transverse" process can be considered independent of $z$, as it noted in the second equation in (10) and justified in [32-34]. Here index $q$ is the counter of such layers. The dependence of $F$ on $z$ is ensured by the fact that the output of each $q^{\text {th }}$ layer is the input of each $(q+1)^{\text {th }}$ layer. In addition to previous assumptions, we also neglect the Rayleigh losses inside the focon since it has quite short length. The parameter $\beta_{0}$ in (10) determines the phase incursion of a wave in a medium with an initially unperturbed refractive index (in the absence of nonlinear processes and any refractive index gradient), i.e. $\beta_{0}=\frac{\omega}{c} n_{0}$. 


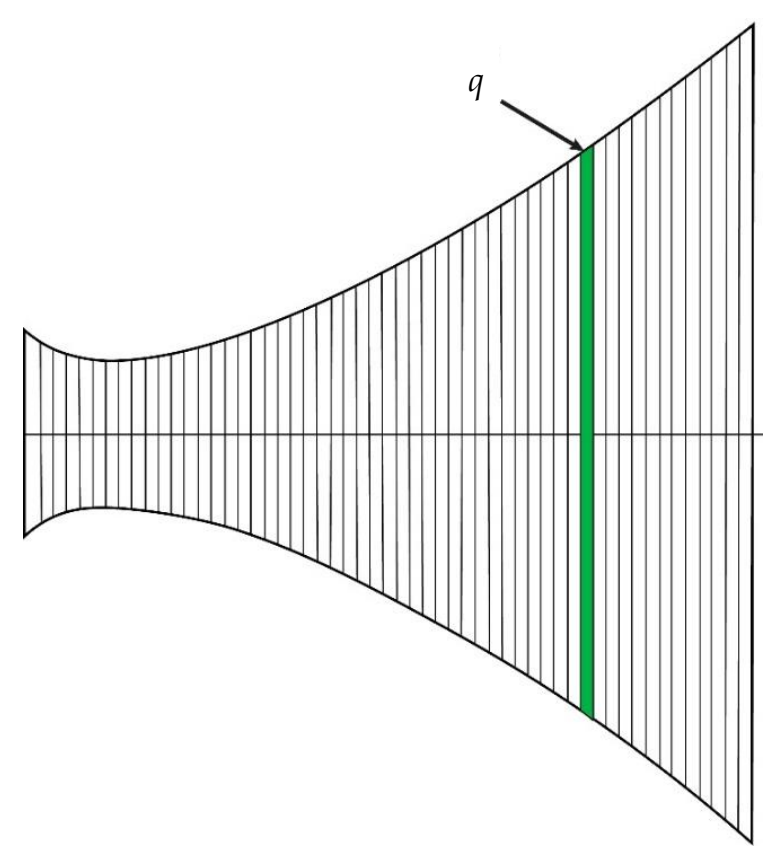

Figure 3. Illustration of the principle of splitting the focon into short layers, $q \in[1, Q], z=\Delta z \cdot q$.

We make the following assumption: there is no re-reflection (and hence interference) between the layers in the longitudinal direction despite the difference in the refractive index between the layers. This is because the focon has a smooth gradient and does not consist of layers with a hop in the refractive index at the boundary. Moreover, when $\Delta z \rightarrow 0$, the increment of the refractive index between the layers is $\Delta n \rightarrow 0$.

Further, if we take into account that in a quadratic-nonlinear medium $\vec{P}_{N L}=\varepsilon_{0} \chi^{(2)} E \cdot \vec{E}$, i.e. $P_{N L}=\varepsilon_{0} \chi^{(2)}|E|^{2}$, and make mathematical transformations similar to the technique [36] for the function $A$, and represent the function $F$ in the form of counterpropagating waves $F_{\uparrow}$ (up) and $F_{\downarrow}$ (down) relative to the focon axis, then for each of the waves (1, 2 and 3$)$ the equations (10) will take a form that is suitable for numerical solution:

$$
\begin{aligned}
& \frac{d A_{1}^{(q)}}{d z}=\varepsilon_{0} \chi^{(2)} \frac{\omega_{1}^{2}}{2 c^{2}}\left[\left(\mu_{11}^{(q)}\left|A_{1}^{(q)}\right|+2 \sum_{m \neq 1} \mu_{1 m}^{(q)}\left|A_{m}^{(q)}\right|\right) A_{1}^{(q)}+2 \mu_{123}^{(q)} A_{2}^{(q) *} A_{3}^{(q)} \exp (j \Delta \beta z) \exp (j \Delta \ell \varphi)\right] \\
& \frac{d A_{2}^{(q)}}{d z}=\varepsilon_{0} \chi^{(2)} \frac{\omega_{2}^{2}}{2 c^{2}}\left[\left(\mu_{22}^{(q)}\left|A_{2}^{(q)}\right|+2 \sum_{m \neq 2} \mu_{2 m}^{(q)}\left|A_{m}^{(q)}\right|\right) A_{2}^{(q)}+2 \mu_{213}^{(q)} A_{1}^{(q) *} A_{3}^{(q)} \exp (j \Delta \beta z) \exp (j \Delta \ell \varphi)\right] \\
& \frac{d A_{3}^{(q)}}{d z}=\varepsilon_{0} \chi^{(2)} \frac{\Omega^{2}}{2 c^{2}}\left[\left(\mu_{33}^{(q)}\left|A_{3}^{(q)}\right|+2 \sum_{m \neq 3} \mu_{3 m}^{(q)}\left|A_{m}^{(q)}\right|\right) A_{3}^{(q)}+2 \mu_{312}^{(q)} A_{1}^{(q)} A_{2}^{(q)} \exp (-j \Delta \beta z) \exp (-j \Delta \ell \varphi)\right] \\
& \frac{d F_{1, \uparrow}{ }^{(l, q)}}{d r}=j\left(\beta_{l}^{(q)}-\beta_{1, r, z}\right) F_{1, \uparrow}{ }^{(l, q)}+j \mathfrak{R}_{1}^{(q)}\left|F_{1, \downarrow}{ }^{(l, q)}\right| \text { and } \\
& \frac{d F_{1, \downarrow}^{(l, q)}}{d r}=j\left(\beta_{l}^{(q)}-\beta_{1, r, z}\right) F_{1, \downarrow}^{(l, q)}+j \Re^{(q)}\left|F_{1, \uparrow}^{(l, q)}\right| \\
& \frac{d F_{2, \uparrow}{ }^{(l, q)}}{d r}=j\left(\beta_{l}^{(q)}-\beta_{2, r, z}\right) F_{2, \uparrow}{ }^{(l, q)}+j \mathfrak{R}_{2}^{(q)}\left|F_{2, \downarrow}{ }^{(l, q)}\right| \text { and } \\
& \frac{d F_{2, \downarrow}{ }^{(l, q)}}{d r}=j\left(\beta_{l}^{(q)}-\beta_{2, r, z}\right) F_{2, \downarrow}{ }^{(l, q)}+j \Re^{(q)}\left|F_{2, \uparrow}{ }^{(l, q)}\right| \\
& \frac{d F_{3, \uparrow}{ }^{(l, q)}}{d r}=j\left(\beta_{l}^{(q)}-\beta_{3, r, z}\right) F_{3, \uparrow}{ }^{(l, q)}+j \mathfrak{R}^{(q)}{ }_{3}\left|F_{3, \downarrow}{ }^{(l, q)}\right| \text { and }
\end{aligned}
$$




$$
\frac{d F_{3, \downarrow}^{(l, q)}}{d r}=j\left(\beta_{l}^{(q)}-\beta_{3, r, z}\right) F_{3, \downarrow}^{(l, q)}+j \Re_{3}^{(q)}\left|F_{3, \uparrow}^{(l, q)}\right|
$$

on each step $\Delta z$ with number $q$. In this case, due to the fact that the function $F$ was divided into two components (up and down), then solution (9) should also take the corresponding form (for each of the waves):

$$
E^{(q)}(r, z, \omega)=\left[\sum_{l=1}^{K_{l}} \tilde{F}_{\uparrow}^{(l, q)}(r) \exp \left(j \beta_{r, z} r\right)+\sum_{k=1}^{K_{l}} \tilde{F}_{\downarrow}^{(l, q)}(r) \exp \left(-j \beta_{r, z} r\right)\right] A^{(q)}(z) \exp \left(-j \beta_{r, z} z\right) .
$$

In equations (11) and (12) we use the following designations: $\mu_{11}, \mu_{22}, \mu_{33}, \mu_{12}$, $\mu_{13}, \mu_{21}, \mu_{23}, \mu_{13}$ and $\mu_{32}$ are the overlap integrals between the corresponding waves ( $\mu_{i v}$ for each pair of $i^{\text {th }}$ and $v^{\text {th }}$ modes), while we assume that $\mu_{12}=\mu_{21}, \mu_{13}=\mu_{31}$ and $\mu_{23}=\mu_{32} ; \mu_{123}, \mu_{213}$ and $\mu_{312}$ - overlap integrals of all three waves: $\mu_{123}=\mu_{213}=\mu_{312}$; parameters $\Delta \beta=\beta_{0,3}+\beta_{0,2}-\beta_{0,1}$, where $\beta_{0, i}$, are determined for the corresponding frequencies $\omega_{i}$ and unperturbed values $n_{0, i}$ at these frequencies. The factor $\exp (j \Delta \ell \varphi)$ in (11) is determined by the difference of the topological charges (an integer) $\ell_{i}$ of the vortex beams for each of the waves and represents the projection of the orbital angular momentum (OAM) on the direction of propagation [1-4]; it is determined by the dependence of the electromagnetic field of the vortex beam on the azimuthal angle $\varphi$. If the waves have no OAM, then the specified multiplier is not taken into account, i.e. $\exp (j \Delta \ell \varphi)=1$ when $\Delta \ell=0$. Otherwise: $\Delta \ell=\ell_{1}-\ell_{2}-\ell_{3}$ by analogy with [1]. The parameter $\beta_{l}^{(q)}$ is the wavenumber of the $q^{\text {th }}$ layer, fig. 3 , which has multipath interference properties. Each layer can be considered as a multibeam interferometer - a Fabry-Perot interferometer (FPI). But due to the fact that the medium is nonlinear, $\beta^{(q)}$ depends on how many re-reflections have already occurred in this $q^{\text {th }}$ layer; here $l$ is the re-reflection counter, $l \in\left[1, K_{l}\right], K_{l}$ in the ideal FPI is equal to $\infty$, but in this case it is determined by the number of effective rereflections and is calculated through the reflection coefficient of the focon profile. For each of the waves, we will assume that:

$$
\begin{gathered}
\beta_{l}^{(q)}=\beta_{M}^{(q)}+\Delta B^{(q)} l, \beta_{M D}^{(q)}=\left.\frac{\pi}{2 \sqrt{f_{1}(\Delta z q)}}\langle n(r, \Delta z q)\rangle\right|_{r}, \\
\Delta B^{(q)}=\frac{2 \pi \chi^{(2)}\left(\sqrt{P^{(q)}}+\sqrt{P^{(q)}}+\sqrt{P^{(q)}}{ }_{3}\right)}{\lambda} \max \left(\mu_{i, m}^{(q)}\right),
\end{gathered}
$$

where $\beta_{M D}^{(q)}$ is the initial (unperturbed) wave number of the $q^{\text {th }}$ FPI with the averaged refractive index over the radius. This leads to the fact that the calculation of $F^{(l, q)}$ is performed for a certain FPI refractive index averaged over the radial gradient, and thus allows one to obtain only an estimations, but, on the other hand, ensures avoidance of the recurrence. Moreover, we apply this approach at the first iteration of the calculation; when the main parameters have already been estimated, in particular, an approximate value $\hat{n}(r, z)$ has been obtained, then for the calculation of $\beta_{M D}^{(q)}$ in (13) it is possible to use the value $\hat{n}(r, z)$ found at the previous iteration. The parameter $\Delta B$ in (13) determines the nonlinear contribution from the transmitted radiation, and is written by analogy with [36, 37], $P_{1}^{(q)}, P^{(q)}{ }_{2}$ and $P_{3}^{(q)}$ are the peak powers of the waves at the point $\Delta z \cdot q$, which at the first iteration can be calculated as $P^{(q)}=\left|E_{0}^{(q)}\right|^{2}$ for each of the waves. The parameter $\beta_{r, z}$ in (12) is defined as $\beta_{r, z}=\frac{\omega \cdot n(r, \Delta z q)}{c}$, but for the first iteration it is determined as 
$\beta_{r, z}=\left.\frac{\omega}{c}\langle n(r, \Delta z \cdot q)\rangle\right|_{r}$. To calculate the coupling coefficient of interfering waves $\mathfrak{R}_{i}, i=1$, 2, 3, similarly to [38], we will use the relation:

$$
\mathfrak{R}_{i}^{(q)} \cong \frac{\pi}{\lambda_{i}}\left|\left(\left.\left\langle n_{i}(r, \Delta z \cdot q)\right\rangle\right|_{r}\right)^{2}-n_{0, i}{ }^{2}\right| \cdot \mu^{(q)}{ }_{i i},
$$

according to which each of the waves interferes only with itself. In (14) the index $i$ of the refractive index relates to the corresponding frequency (wavelength).

The number of effective re-reflections in the FPI can be determined as [39, 40]: $K_{l}=\frac{\pi \cdot \sqrt{\rho_{z}}}{\left(1-\rho_{z}\right)}$ where $\rho^{(q)}$ is the reflection coefficient of the focon profile at arbitrary point $z$ (due to reflection from the interface between media with different refractive indices): $\rho^{(q)}=\frac{\left|n\left(r=\sqrt{f_{1}(\Delta z \cdot q)}, z\right)-n_{\mathrm{cl}}\right|}{n\left(r=\sqrt{f_{1}(\Delta z \cdot q)}, z\right)+n_{\mathrm{cl}}}$. The overlap integrals $\mu$ for the considered waves (for each pair of $i$-th and $v$-th modes) are determined by analogy with [36]:

$$
\mu_{i, v}^{(q)}=\frac{\left\langle\left(F_{\uparrow, i}^{(q)}+F_{\downarrow, i}^{(q)}\right)\left(F_{\uparrow, v}^{*(q)}+F_{\downarrow, v}^{*(q)}\right)\right\rangle}{\sqrt{\prod_{i}\left\langle\left|F_{\uparrow, i}^{(q)}+F_{\downarrow, i}^{(q)}\right|^{2}\right\rangle}},
$$

where the terms $F_{\uparrow, i}^{(q)}$ and $F_{\downarrow, i}^{(q)}$ for each of the waves can be found using the properties of the FPI for re-reflecting (superposing) waves [38]:

$$
\begin{gathered}
F_{\uparrow}{ }^{(q)}=\sum_{l=1}^{K_{l}+1}\left(\rho^{(q)}\right)^{l-1}(\Delta z \cdot q) F_{\uparrow}^{(0, q)} \exp \left(j(l-1) \delta^{(q)}\right) ; \\
F_{\downarrow}^{(q)}=\sum_{l=1}^{K_{1}+1}\left(\rho^{(q)}\right)^{l-1}(\Delta z \cdot q) F_{\downarrow}^{(0, q)} \exp \left(j(l-1) \delta^{(q)}\right) ; F^{(q)}=F_{\uparrow}^{(q)}+F_{\downarrow}^{(q)} .
\end{gathered}
$$

The parameter $\delta$ will be defined by analogy with $\delta^{(q)}=\left.\frac{4 \pi \cdot \sqrt{f_{1}(\Delta z \cdot q)}}{\lambda} \cdot\langle n(r, \Delta z \cdot q)\rangle\right|_{r}$, and the initial amplitude value will be set for the first iteration as: $F_{\uparrow}^{(0, q)}=F_{\downarrow}^{(0, q)} \cong \frac{1}{4} \sqrt{P^{(q)}}$, assuming that the refraction index is isotropic within the layer and therefore the power is divided equally, i.e. a half for each wave. In addition, for the components of the terms $F$ under consideration, which characterize re-reflections from the focon profile in the transverse direction, the following one can use the following relations:

$$
\frac{F_{\downarrow}^{(l, q)}}{F_{\uparrow}^{(l+1, q)}}=\rho^{(q)}=\frac{F_{\uparrow}^{(l, q)}}{F_{\downarrow}^{(l+1, q)}}, F_{\downarrow}^{\left(K_{1}, q\right)}=F_{\downarrow}^{(0, q+1)}, F_{\uparrow}^{\left(K_{1}, q\right)}=F_{\uparrow}^{(0, q+1)} .
$$

The transformation (8)-(11) is generally known, so we considered it unnecessary to present a detailed derivation of equations (11) in the article. Let us pay attention to the fact that the left parts of each equation for the functions $A$ in (11) do not depend on the angle $\varphi$, so the solutions of system (11) exist under the condition: $\Delta \ell=0$. This suggests that in a quadratic nonlinear medium the input signals carrying OAMs $\ell_{1}$ and $\ell_{2}$ at frequencies $\omega_{1}$ and $\omega_{2}$ generate an output signal with OAM $\ell_{3}=\ell_{1}-\ell_{2}$ and frequency $\omega_{3}=\omega_{1}-\omega_{2}$, which ensures the seamlessness of optical-radio engineering conversion. Note that for the existence of OAM modes, as is well known [31], a few-mode regime (excitation of several transverse modes) is required. That's why we search $n(r, z)$ and $f_{1}(z)$ - to provide 
proper few-mode regime. Therefore, we reckon that the wave field (for each of the waves under consideration), determined by (12), should obey the following relation:

$$
E_{M-1}^{(q)}=\sqrt{P^{(q)}} \frac{J_{\ell}\left(u_{\ell m} \frac{r}{\sqrt{f_{1}(\Delta z \cdot q)}}\right)}{J_{\ell}\left(u_{\ell m}\right)} \cdot e^{-j \beta^{*} z} e^{-j \omega t} e^{\ell \varphi},
$$

where $J_{\ell}$ is the Bessel function of the $\ell$-kind, $u \in m$ is the first $\ell m^{\text {th }}$ maximum of the $J_{\ell}, P^{(q)}$ is the power parameter, and $\beta^{*}$ is the generalized mode propagation coefficient. The specified "quasi-single-mode" addition will ensure the absence of dark areas, which means the large volume of the interacting light, and with this the highest efficiency of the device (especially in case of low-order OAMs, e.g., $\ell_{1}=1, \ell_{2}=0$, etc.). Relation (18) is valid for $E$ as a function of time, to which inverse Fourier transform (12) should be performed.

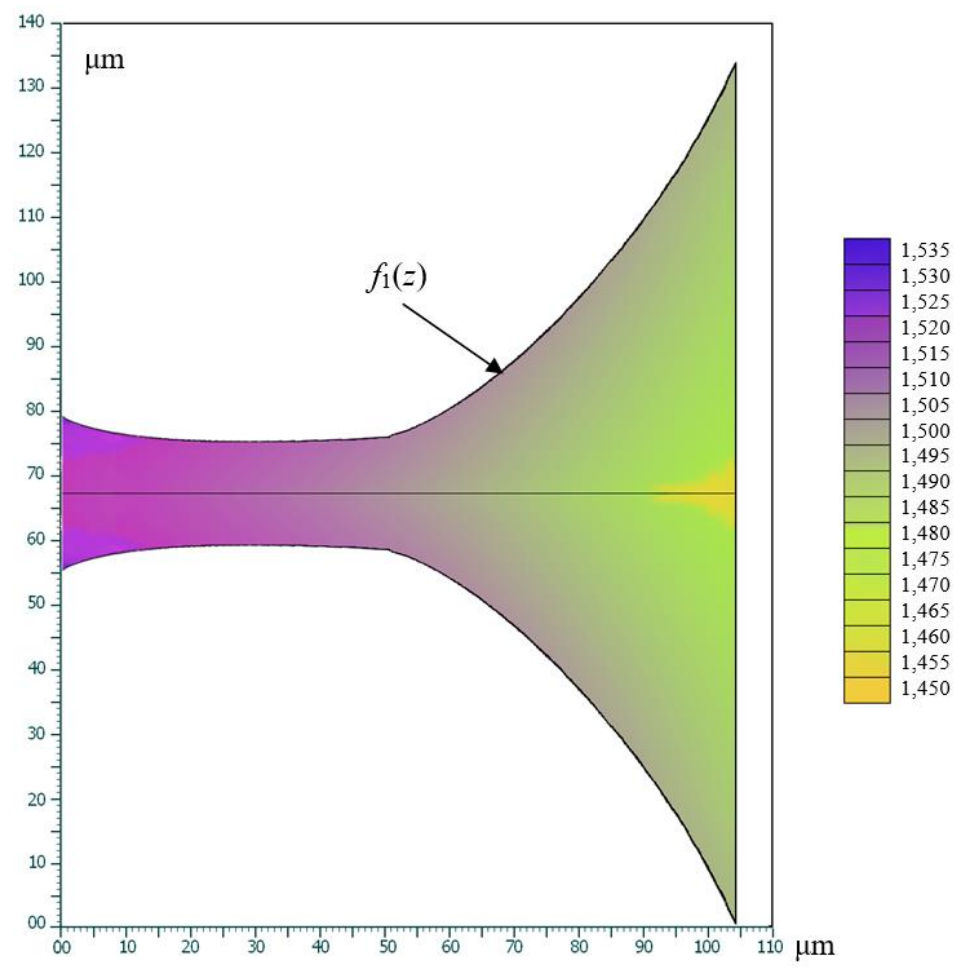

Figure 4. An illustration of the found parameters and at the second iteration of the calculation, providing the power of the generated wave $P_{3}$ is at least $1 \%$ of $P_{1}$.

Note that the stated condition can be simplified. Namely: the power parameter in (18) can be considered only as a multiplier i.e. as weight coefficient. In addition, the considered amplitude distribution has no dependence on $z$ (except the phase exponent), but only on $r, \varphi$, so condition (18) applies only to function $F$. Therefore, we consider that relation (18), which is valid for $F^{(q)}$ as a function of time, complements relations (16). As a result of the numerical solution of equations (11), taking into account (13)-(18), iterative selection of the functions $n(r, z)$ and $f_{1}(z)$ was carried out, fig. 4. In calculations we assumed $\chi^{(2)}=10^{-6} \mathrm{~m} / \mathrm{V}$. As we can see in fig. 4 , the proposed converter structure can be easily coupled with few-mode fiber due to a comparative input diameter of a few microns.

\section{Conclusions}

In this article we provide an analysis of currently known materials and structures with quadratic-nonlinear optical properties. These materials and structures can be used in the process of non-linear parametric generation of the difference frequency in a twofrequency optical-radio engineering conversion. 
The principle of constructing a nonlinear optical-radio-technical converter based on optical focon is proposed. Using the assumption that this focon can be made by MOF, a technique for modeling its parameters is proposed. The mathematical model of the process of propagation and nonlinear interaction of waves inside the focon is based on a simplification of the nonlinear wave equation.

Within the framework of the developed model, the following parameters are approximately determined: the 3D gradient of the linear refractive index and the function characterizing the geometric profile of the focon, which provide quasi-single-mode generation of the difference frequency. The proposed scheme can be successfully used in the deployment of fiber-optic-radio communication network segments with seamless optical-radio conversion (including vortex-signal conversion), which will greatly increase the capacity and throughput of telecommunication systems.

Author Contributions: Conceptualization, formal analysis and methodology: I.L.V., A.R.G.; funding acquisition, formatting, I.K.M., A.V.B.; software and visualization, I.L.V.; writing - original draft preparation, review and editing, I.L.V., A.R.G, I.K.M., A.V.B., M.T. All authors have read and agreed to the published version of the manuscript.

Funding: This work was partially supported by the Ministry of Science and Higher Education of the Russian Federation for research under the State Assignment of FSBEI HE USATU No. FEUE2020-0007 on the topic "Theoretical foundations of modeling and semantic analysis of the processes of transformation of vortex electromagnetic fields in infocommunication systems" in the part "Analysis of optical quadratic-nonlinear materials and structures potentially suitable for the design of a nonlinear converter" and partially funded by RFBR, DST, NSFC and NRF according to the research project 19-57-80016 BRICS_t in the part "Modeling the parameters of the proposed device".

Conflicts of Interest: The authors declare no conflict of interest.

\section{References}

1. Bagmanov, V.; Sultanov, A.; Gizatulin, A.; Meshkov, I.; Kuk, I.; Grakhova, E.; Abdrakhmanova, G.; Vinogradova, I. Optics-toTHz conversion of vortex beams using nonlinear difference frequency generation. Comput. Opt. 2019, 43, $983-991$.

2. Bagmanov, V.H.; Sultanov, A.K.; Meshkov, I.K.; Gizatulin, A.R.; Nigmatullin, R.R.; Sakhabutdinov, A.Z. Propagation and Transformation of Vortexes in Linear and Nonlinear Radio-Photon Systems. Fibers. 2022, 10 , 4. https://doi.org/10.3390/fib10010004.

3. Meshkov, I.; Gizatulin, A.; Vinogradova, I.; Meshkova, A; Sultanov, A.; Bagmanov, V.; Bourdine, A. Usage of SDM technology in radio-over-fiber (RoF) transmission systems in high-speed scalable 6G wireless networks. Proc. SPIE 11793, Optical Technologies for Telecommunications 2020. 2021, 117931G. doi:10.1117/12.2593036.

4. Vinogradova, I.; Gizatulin, A.; Meshkov, I.; Bagmanov, V.; Morozov, O.; Gabdulkhakov, I.; Ganchevskaya, S.; Kazanskiy, N.; Sultanov, A. Influence of Two-Frequency Radiation Intensity Fluctuations on the Output Signal of a Vortex Optical Fiber Forming OAM Address in Polyharmonic Sensor Technology. Photonics. 2021, 8, 351. https://doi.org/10.3390/photonics8090351

5. Miyamoto, K.; Sano, K.; Miyakawa, T.; Niinomi, H.; Toyoda, K.; Vallés, A.; Omatsu, T.; Generation of high-quality terahertz OAM mode based on soft-aperture difference frequency generation. Opt. Exp. 2019, 27(22):31840. DOI:10.1364/OE.27.031840.

6. Zhang, I.; Zhao, X.; Zheng, Y.; Li, H.; Chen, X. Universal modeling of second-order nonlinear frequency conversion in threedimensional nonlinear photonic crystals. Opt. Exp. 2018, Vol. 26, Issue 12, pp. 15675-15682. https://doi.org/10.1364/OE.26.015675.

7. Suizu, K.; Shibuya, T.; Nagano, Sh.; Akiba, T.; Edamatsu, K.; Ito, H.; Kawase, K.; Pulsed High Peak Power Millimeter Wave Generation via Difference Frequency Generation Using Periodically Poled Lithium Niobate. Japanese Journal of Applied Physics. 2007, Vol. 46, No. 40, 2007, pp. L982-L984.

8. Wei, D.; Wang, Ch.; Wang, H.; Hu, X.; Wei, D.; Fang, X.; Zhang, Y.; Wu, D.; Hu, Y.; Li, J.; Zhu, Sh.; Xiao, M. Experimental demonstration of a three-dimensional lithium niobate nonlinear photonic crystal. Nature Photonics. 2018, 12, pp. 596-600. https://doi.org/10.1038/s41566-018-0240-2.

9. Sungwon, K. Optical, electrical and elastic properties of ferroelectric domain walls in LiNbO3 and LiTaO3, A Thesis in Materials, 2003; p. 230.

10. Zhabotinskiy, M.E. Quantum electronics; Physical Encyclopedia: Vol.2, Moscow, Russia, 1990; pp. 319-320.

11. Koch, S. W.; Peyghambarian, N.; Gibbs H. M. Band-edge nonlinearities in direct-gap semiconductors and their application to optical bistability and optical computing. Journal of Applied Physics. 1988, 63 R1. https://doi.org/10.1063/1.340098.

12. Joannopoulos, J.D.; Johnson, S. G.; Winn, J. N.; Meade, R. D. Photonic Crystals: Molding the Flow of Light: Princeton University press, 2008; $283 \mathrm{p}$.

13. Jazbinsek, M.; Puc, U.; Abina, A.; Zidansek, A. Organic Crystals for THz Photonics. Appl. Sci. 2019, 9(5), 882. https://doi.org/10.3390/app9050882 
14. Marder, S.; Tiemann, B.; Perry, J. W. New Organic and Organometallic Salts For Second-Order Nonlinear Optics. Proc. SPIE 1147, Nonlinear Optical Properties of Organic Materials II, 1990. https://doi.org/10.1117/12.962113.

15. Calabrese, J. C.; Lap-Tak, Ch.; Green, J. C.; Marder, S. R.; Tamt, W.; Molecular Second-Order Optical Nonlinearities of Metallocenes. J. Am. Chem. SOC. 1991, 113, pp. 7227-7232.

16. Hierlinger, C.; Cordes, D. B.; Slawin, A.; Colombo, A.; Dragonetti, C.; Righetto, S.; Roberto, D.; Jacquemin, D.; Zysman-Colman E.; Guerchais, V. An investigation on the second-order nonlinear optical response of cationic bipyridine or phenanthroline iridium (III) complexes bearing cyclometallated 2-phenylpyridines with a triphenylamine substituent. Dalton Trans. 2018, 47(25), pp. 8292-8300. DOI:10.1039/C8DT00754C.

17. Mendiratta, Sh.; Lee, Ch.-H.; Usman, M.; Lu, K.-L.; Metal-organic frameworks for electronics: emerging second order nonlinear optical and dielectric materials. Sci. Technol. Adv. Mater. 2015, 16, 054204 (7pp). doi:10.1088/1468-6996/16/5/054204.

18. Mayer, S.A. Plasmonics: theory and applications; Izhevsk: Research Center "Regular and Chaotic Dynamics", $2011 .-296$ p.

19. Zhang, L.; Li, H.; He, H.; Yang, Y.; Cui, Y.; Qian, G. Structural Variation and Switchable Nonlinear Optical Behavior of MetalOrganic Frameworks. Nano-micro small. 2021, Volume 17, Issue 6, 2006649. https://doi.org/10.1002/smll.202006649.

20. Huang, J.; Li, T.; Liu, Y.; Yang, K.; Feng, T.; Liang, Y.; Zhao, Y.; Song, Y.; Chu, H.; Zhao, Sh.; Li, D.; Qiao, W.; Broadband and enhanced nonlinear optical modulation characteristics of CuBTC for pulsed lasers. Optical Materials Express. 2021, Vol. 11, Issue 10, pp. 3546-3555. https://doi.org/10.1364/OME.437408.

21. Li, H.; Zhang, L.; He, H.; Yang, Y.; Cui, Y.; Qian, G. Tunable nonlinear optical responses based on host-guest MOF hybrid materials. Science China Materials. 2021, Vol. 64, pp. 698-705. DOI:10.1007/s40843-020-1455-6.

22. Pravini, M. G.; Fernandoa, S.; Wijewardena Gamalathb, K. A. I. L. Nonlinear optical properties of photonic crystals. World Scientific News. 2018, 97, pp. 1-27.

23. Tzankov, P.; Petrov, V. Effective second-order nonlinearity in acentric optical crystals with low symmetry. Applied Optics. 2005, Vol. 44, No. 32, pp. 6971 - 6985. DOI: 10.1364/AQ.44.006971.

24. Feigel, A.; Kotler, Z.; Sfez, B. Artificial Second Order Non-Linearity in Photonic Crystals. Mat. Res. Soc. Symp. Proc. 2011, Vol. 694, pp. 709-713. DOI:10.1557/PROC-694-K9.5

25. Dumeige, Y.; Sagnes, I.; Monnier, P.; Vidakovic, P.; Abram, I.; Mériadec, C.; Levenson, A. Phase-Matched Frequency Doubling at Photonic Band Edges: Efficiency Scaling as the Fifth Power of the Length. Phys. Rev. Lett. 2002, 89, 043901.

26. Centini, M.; Sibilia, C.; Scalora, M.; D’Aguanno, G.; Bertolotti, M.; Bloemer, M. J.; Bowden, C. M.; Nefedov, I. Dispersive properties of finite, one-dimensional photonic band gap structures: Applications to nonlinear quadratic interactions. Phys. Rev. 1999, E 60, 4891.

27. D’Aguanno, G.; Centini, M.; Scalora, M.; Sibilia, C.; Dumeige, Y.; Vidakovic, P.; Levenson, J. A.; Bloemer, M. J.; Bowden, C. M.; Haus, J. W.; Bertolotti, M. Photonic band edge effects in finite structures and applications to $\chi(2)$ interactions. Phys. Rev. 2001, E 64, 016609.

28. Yao; Y.; Knox, W. H. Difference frequency generation of femtosecond mid infrared pulses employing intense Stokes pulses excitation in a photonic crystal fiber. Optics Express. 2012, (20)23, pp. 25275-25283. https://doi.org/10.1364/OE.20.025275.

29. Tao, Ch.; Junqiang, S.; Linsen, L.; Jianguan, T.; Yujie, Z.Design of a Photonic Crystal Waveguide for Terahertz-Wave DifferenceFrequency Generation. IEEE Photonics Technology Letters, 2012, 24(11), pp. 921-923. DOI:10.1109/LPT.2012.2190890.

30. Meshkov, I.K.; Gizatulin, A.R.; Kuk, I.A.; Grakhova, E.P.; Abdrakhmanova, G.I.; Voronkova, A.V.; Vinogradova, I.L.; Bagmanov, V.K.; Sultanov, A.K. The Principle of Constructing Antenna Arrays for Generating OAM Radio Waves Based on Multipole Decomposition of Electromagnetic Field. 26th Telecommunications Forum, TELFOR. 2018, 8611889. DOI: 10.1109/TELFOR.2018.8611889.

31. Kotlyar, V.V.; Kovalev, A.A.; Porfirev, A.P. Vortex Laser Beams; CRC Press: Boca Raton, FL, USA, 2018.

32. Sultanov, A.Kh.; Vinogradova, I.L.; Meshkov, I.K.; Andrianova, A.V.; Abdrakhmanova, G.I.; Ishmiyarov, A.A.; Yantilina, L.Z. A method for connecting antenna radiators to RoF systems using an optical device and calculating its parameters. Computer Optics. 2015, 39(5), pp. 728-37. DOI: 10.18287/0134-2452-2015-39-5-728-737.

33. Vinogradova, I.L.; Salikhov, A.I.; Meshkov, I.K.; Gizatulin, A.R.; Sultanov, A.K.; Bagmanov, V.K. Analyzing the optical modes and vortex signals in an optical fiber by means of side illumination. Journal of Optical Technology. 2021, (88)10, pp. 597-605. https://doi.org/10.1364/JOT.88.000597.

34. Gizatulin, A.R.; Meshkov, I.K.; Vinogradova, I.L.; Bagmanov, V.K.; Sultanov, A.K. Chiral fiber Bragg gratings for vortex signal generation. Proc. SPIE 11793, Optical Technologies for Telecommunications 2020. 2021, 117930A. DOI: 10.1117/12.2593041.

35. Vinogradova, I.L.; Salikhov, A.I.; Meshkov, I.K.; Gizatulin, A.R.; Sultanov, A.K.; Bagmanov, V.K.; Zhitnikov, V.P. Method of non-destructive instrumental analysis of mode compositions and vortex signals in guiding structures. Proc. SPIE 11793, Optical Technologies for Telecommunications 2020. 2021, 117930M. DOI:10.1117/12.2593039.

36. Agrawal, G.P. Applications of Nonlinear Fiber Optics; Academic Press: Cambridge, MA, USA, 2008; p. 508.

37. Gizatulin, A.R.; Meshkov, I.K.; Vinogradova, I.L.; Bagmanov, V.K.; Grakhova, E.P.; Sultanov, A.K. Generation of Vortex Optical Beams Based on Chiral Fiber-Optic Periodic Structures. Sensors. 2020, 20(18), 5345. https://doi.org/10.3390/s20185345.

38. Kivshar, Y.S.; Agrawal, G.P. Optical solitons. From optical fibers to photonic crystals; FIZMATLIT: Moscow, $2005 ;$ p. 648.

39. Matveev, A.N. Optics; Higher school: Moscow, 1985; p. 351.

40. Hui, R.; O'Sullivan, M. Chapter 2 - Basic Instrumentation for Optical Measurement; Fiber Optic Measurement Techniques, Academic Press, 2009; pp. 129-258. 